\title{
Temporal and interactional effects in short-term storage'
}

HERMAN BUSCHKE AND HOWARD LIM ${ }^{2}$

STANFORD UNIVERSITY
The operation of temporal and interactional processes in short-term storage was studied under conditions which require that information about all items presented be stored and the relative retention of all be evaluated concurrently, but preclude further interference after presentation by testing or retrieval effects. Random sequences of twelve two digit numbers from a known set of thirteen were presented visually at 1,2 , or 4 per sec. for full interval and $.25 \mathrm{sec}$. presentation durations to 15 Ss who reported the missing number that was not presented from the set. For $1 / \mathrm{sec}$. full duration presentations the missing scan serial position error distribution shows a linear decrease in errors from first through last presented items. As rate of presentation increases there is a linear increase in total errors, a linearly increasing primacy effect, and an increase of errors over at least the last half of the sequence such that the error distribution remains linear with the same slope. These findings are compatible with the operation of time dependent interactional and perceptual processes in short-term storage but not with autonomous decay.

The purpose of this study is to determine whether temporal processes affect short-term storage (STS) in the missing scan (Buschke, 1963a), and if so, whether directly (decay) or through effects on interactional processes (interference). This is of interest because the missing scan may more clearly demonstrate processes operating in storage since it requires retention of information about all items presented while at least minimizing retrieval effects.

Temporal effects in short-term memory (STM) have been studied by various methods, with conflicting results (Brown, 1958; Conrad \& Hille, 1958; Corballis, 1966; Crowder, 1966; Mackworth, 1962; Norman, 1966; Peterson, 1966; Waugh \& Norman, 1965). Two sources of difficulty in some studies of this problem may be restriction of retention and retrieval to some rather than all presented items, and possible confounding of temporal and interactional effects in retrieval with those in storage. Thus it would seem useful to obtain information about relative retention in STS of sequentially presented events in a situation which requires retention of all presented items and evaluates the relative retention of all concurrently, but is free of retrieval effects.

The missing scan paradigm is based on the S's knowledge of the membership of a set: after presentation of all but one member of the set in random sequence, the $S$ indicates the missing member. For example, when $5,3,6,4,1$ are presented from the set 1-6, the missing number is 2 . Since Ss presumably must retain all items presented to choose the missing one correctly, this paradigm seems to satisfy the assumption necessary for any estimation of storage capacity, namely that the $S$ will attempt to store all items presented. The missing scan has provided data which may be interpreted as reflecting an overall STS capacity of at least 10 items (Buschke, 1963b): on the average, $33 \%$ of the time the missing number was chosen when 12 numbers from a set of 13 were presented, which is equivalent to retention of 10 items with guessing among the remaining three alternatives.

When a $S$ fails to choose the missing item, he must report as missing some item actually presented (an error of commission). Such errors should reflect the relative retention of items immediately after presentation (without further interference), since all items must be retained and the choice of the missing item should require concurrent evaluation of all items. In other words, relative retention of all presented items may be estimated because, in effect, the least well remembered item should be chosen when such an error is committed. Thus a more adequate delineation of interactional effects also should be achieved because this method requires retention and subsequent choice among all items presented. For auditory presentation at about $1 / \mathrm{sec}$. the missing scan serial error curve generated by such errors has shown a generalized decrease of errors from second through most recently presented items, with a primacy effect (Buschke, 1963b). This report describes systematic changes in total errors and their serial position distribution as rate of visual presentation increases, and considers their implication for understanding STS.

\section{Method}

Random sequences of 12 numbers from 13-25 inclusive were presented visually by an I.E.E. Binavue preprogrammed by teletype. The $\mathrm{Ss}$, who were $\mathbf{1 5}$ male college students paid for their participation, were instructed to indicate the missing number at the end of each sequence by pressing the appropriate button on a circular response panel.

Lists of 12 numbers randomly chosen from the set of 13 were presented sequentially at rates of $1 /$ sec., $2 / \mathrm{sec}$, or $4 / \mathrm{sec}$., for either the full interval before the next item or for only .25 sec., making five conditions of presentation: $1 / \mathrm{S}-\mathrm{F}, 1 / \mathrm{S}-\mathrm{P}, 2 / \mathrm{S}-\mathrm{F}, 2 / \mathrm{S}-\mathrm{P}$, and $4 / \mathrm{S}$ (where $\mathrm{F}=$ full interval, and $\mathrm{P}=$ partial interval or $.25 \mathrm{sec}$. presentation). In each condition there were 


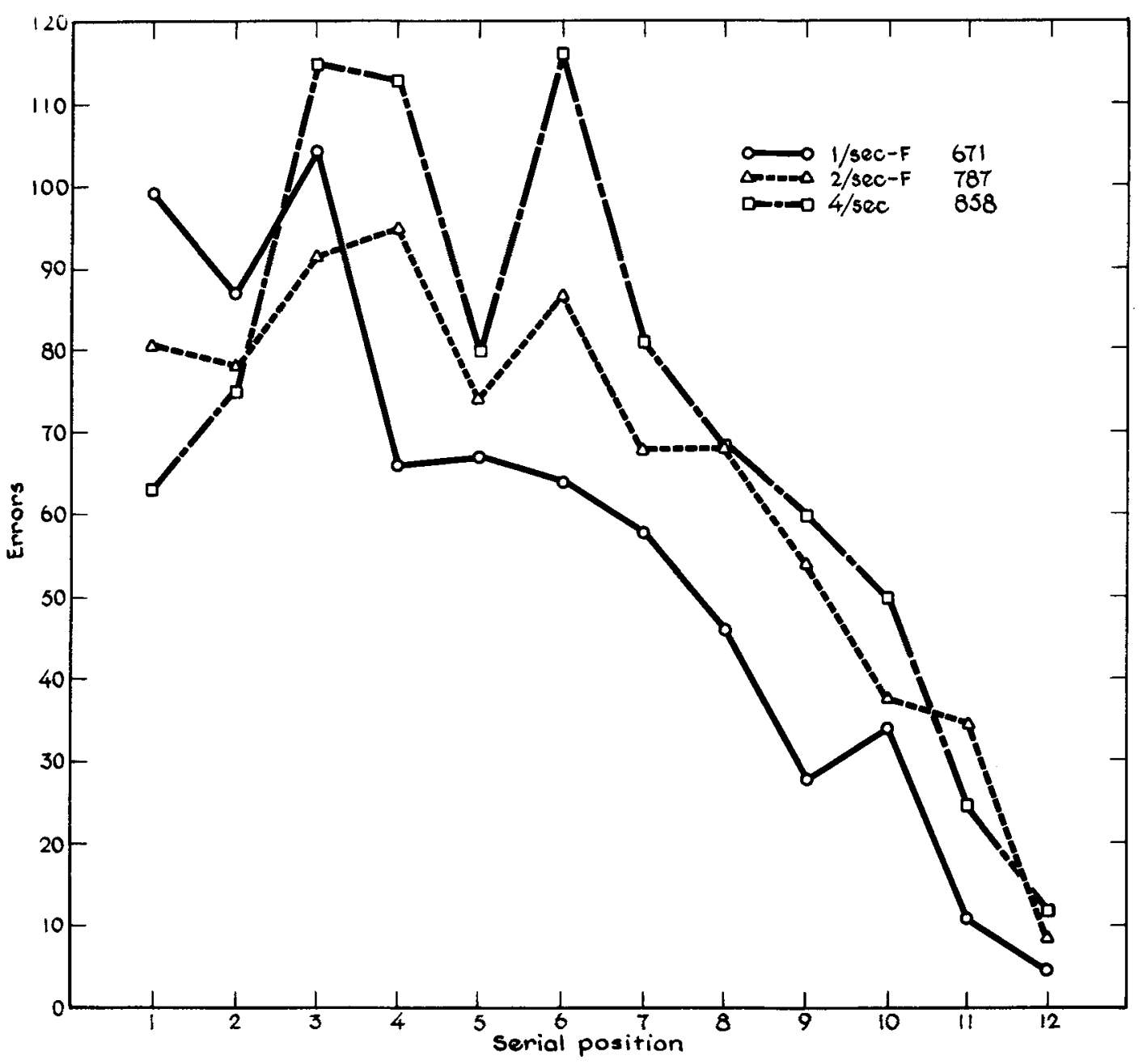

Fig. 1. Serial position error distributions for full interval presentations at 1, 2, and 4/sec. presentation rates. Total errors in key.

78 lists so designed that all numbers appeared in each serial position and were missing items equally often at random. All lists of any condition were presented in a block, the order of conditions being counterbalanced across Ss.

\section{Results}

Figures 1 and 2 show the number of errors made by all Ss as a function of serial position for each condition, with the total number of errors given in the key. Performance is well above chance in all conditions, ranging from $43 \%$ correct at $1 / \mathrm{S}-\mathrm{F}$ to $26 \%$ correct at $4 / \mathrm{S}$, which yield estimates of overall retention between 11 and 9 items. This is consistent with the earlier missing scan estimate of at least 10 item capacity for STS (Buschke, 1963b).

The total number of errors increases linearly with increasing rate of presentation for both full and partial (.25 sec.) presentation (linear trend components sig- nificant, $.01<\mathrm{p}<.05$, for correlated observations). Analysis of variance of total errors showed significant overall differences $(F=4.49$, df $=4 / 44, p<.01)$. Selected contrasts showed that $4 / \mathrm{S}>1 / \mathrm{S}-\mathrm{F}(\mathrm{p}<.001), 4 / \mathrm{S}>$ $1 / \mathrm{S}-\mathrm{P} \quad(\mathrm{p}<.001), 2 / \mathrm{S}-\mathrm{F}>1 / \mathrm{S}-\mathrm{F} \quad(\mathrm{p}<.001)$ (McNemar, pp. 285-287). Differences between full and partial (.25 sec.) duration of presentation were not significant for either $1 / \mathrm{sec}$. or $2 / \mathrm{sec}$. rate of presentation.

The distribution of errors by serial position in the $1 / \mathrm{S}-\mathrm{F}$ condition describes a linear decrease of errors from first through most recently presented items $(y=66.7-10.4 X$, linear trend analysis significant at $p<.001)$. As rate of presentation increases, the serial position error distribution shows (i) an increase of errors such that the function remains linear with the same slope, at least over serial positions $7-12$, together with (ii) an increasing primacy effect manifested by fewer errors in serial positions 1 and 2 (Figs. 1 and 2). Analysis of variance of the errors over serial 
positions 7-12 showed significant differences $(F=15.8$, $\mathrm{df}=4 / 20, \mathrm{p}<.001)$. The following selected contrasts of number of errors were significant (McNemar, pp. 285-287): $1 / \mathrm{S}-\mathrm{F}<2 / \mathrm{S}-\mathrm{F}, 1 / \mathrm{S}-\mathrm{F}<4 / \mathrm{S}, 1 / \mathrm{S}-\mathrm{P}<2 / \mathrm{S}-\mathrm{F}$, $1 / \mathrm{S}-\mathrm{P}<4 / \mathrm{S}$ at $\mathrm{p}=.01$ and $1 / \mathrm{S}-\mathrm{P}<2 / \mathrm{S}-\mathrm{P}, 1 / \mathrm{S}-\mathrm{F}<$ $2 / \mathrm{S}-\mathrm{P}$ at $\mathrm{p}=.05$. Examination of the error curves over the last six positions (7-12) shows that for each of the cirves a significant linear component is present (p<.01 (McNemar, pp 285-287)). No significant differences were found among the slopes of the linear components over serial positions 7-12 for these conditions. A linear increase in primacy effect with increasing rate of presentation is shown for serial positions 1 and 2 with full duration presentations by significant negative slopes of the decrease in proportion of errors as rate of presentation increases ( $F=$ 22.4, $\mathrm{df}=1 / 28, \mathrm{p}<.001$, and $\mathrm{F}=4.75, \mathrm{df}=1 / 28, \mathrm{p}<.05$ respectively (McNemar, pp. 351-352)). No difference between the error distributions of full or partial
$2 /$ sec. presentations are apparent, while at $1 / \mathrm{sec}$. presentation rate, the only difference between full and partial error distributions appears as a primacy effect seen only in the latter.

These results are illustrated by Fig. 3, which shows the number of errors occurring in each quarter of the sequential presentation. The data were put in this form for evaluation of overall differences in error distributions by testing selected contrasts between quarters by the Scheffe' method, which showed that comparison of quarters 1 with 2 and of quarters 1 with 3 confirms significant differences between $1 / \mathrm{S}-\mathrm{F}$ and $2 / \mathrm{S}-\mathrm{F}(\mathrm{p}<.01)$ and between $1 / \mathrm{S}-\mathrm{F}$ and $4 / \mathrm{S}$ $(p<.01)$.

In summary, this study of temporal effects in shortterm storage shows the following. (i) There is a linear increase in total errors as the rate of presentation increases. (ii) A linear decrease in errors from first through last serial positions occurs at

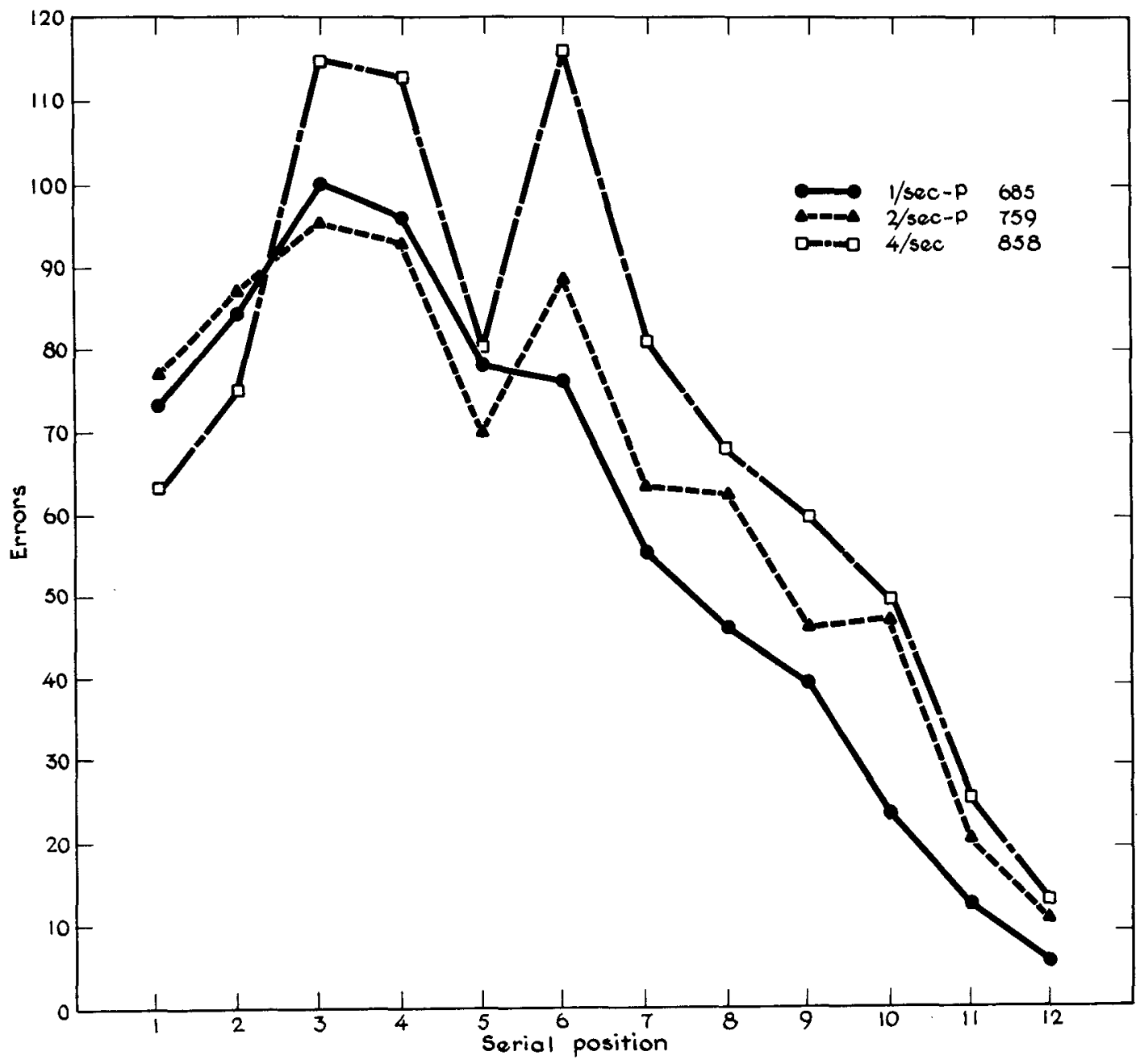

Fig. 2. Serial position error distributions for partial interval (.25 sec.) presentations at 1,2 , and $4 /$ sec. presentation rates. Total errors in key. 


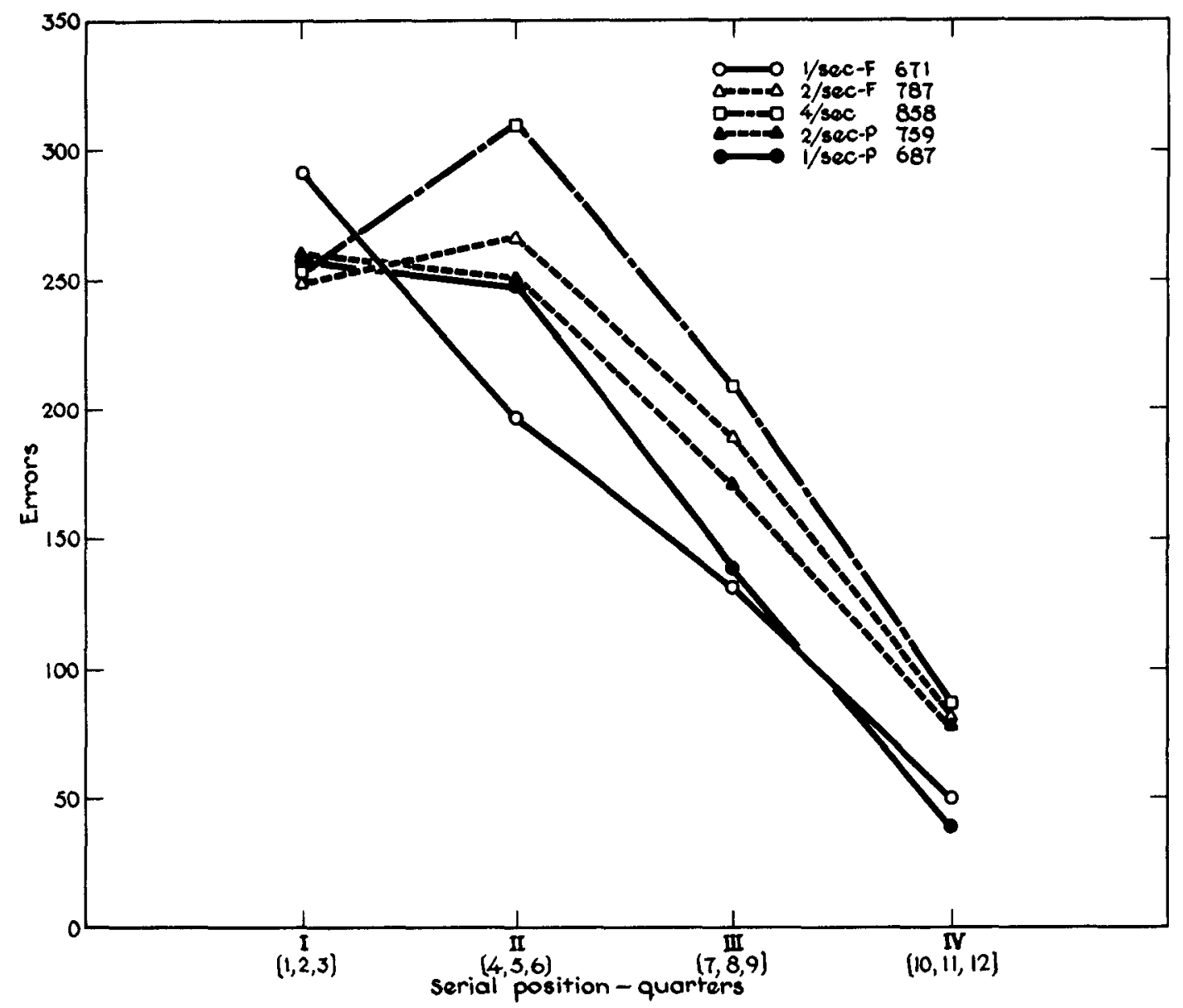

Fig. 3. Serial position error distributions by quartiles for full and partial (.25 sec.) presentations at 1,2 , and $4 /$ sec. presentation rates. Total errors in key.

full curation $1 / \mathrm{sec}$. presentation. (iii) There are systematic changes in the serial position error distribution with an increasing rate of presentation. (a) There is a linear decrease in the proportion of errors occurring in serial positions 1 and 2 with full duration presentations (linearly increasing primacy effect). (b) More errors occur elsewhere, at least over serial positions 7-12, where the error distribution remains linear with the same slope. (iv) There are no significant differences between full and partial interval presentation duration for either the total errors or the overall distribution of errors.

\section{Discussion}

Clearly, there are temporal effects in short-term memory (STM), shown by systematic changes in total errors and their distribution according to serial position as rate of presentation increases in the missing scan. If, as seems reasonable, the missing scan method used in this study is free of retrieval effects, then these effects occur in short-term storage (STS), and consideration of factors affecting such temporal changes may reasonably be restricted to those affecting entry into and retention in STS. Potentially relevant factors might Include the following presumably non-exhaustive list:

"Clearing" of STS, by (a) processes operating naturally in STS such as decay or interference, or (b) turning off some normal renewal processes which otherwise counteract natural clearing processes, or (c) some active erasure process.

Perception and encoding, which may not be distinct processes if what is encoded is that some specific perception occurred. For example, in the missing scan, perception might consist of a match between currently relevant alternatives and what is presented, the match itself being the form in which information that the item occurred is encoded. It may be pertinent to distinguish types of perception in terms of their function, since it seems reasonable that what is perceived about an event (i.e., how it is perceived) will depend on how that perception is to be used (i.e., what its anticipated function is). Perception of an event may well differ both quantitatively 
and qualitatively in terms of number and kinds of criterial attributes perceived depending on whether one is to identify that event or remember it, and in the latter case, whether by recall or recognition. If this view is correct, it raises problems about evaluation of perception in memory studies, since, for instance, the kind of perception adequate for writing down a presented item at time of presentation may not be the kind of perception necessary for remembering that item in order to write it later. Perception will be used in this discussion to mean perception relevant for encoding and storage. This suggests that perception and encoding may be affected by interactional factors such as storage load and interference. While proactive interference (PI) by earlier items might affect either or both the perception and encoding, as well as the retention of later items, retroactive interference (RI) by later items can affect only the retention and recoding but not the perception and initial encoding of earlier items. Temporal effects on perception and encoding might be independent (as in perseveration and consolidation of an independent trace) or secondary to their effects on interactional processes such as PI.

Renewal processes, of which rehearsal may be only one kind. Such renewal processes would be relevant only if some other process such as decay, interference or recoding, leading to loss or decreased accessibility of information, also operated in STS. Renewal processes would seem most relevant to decay, since enough relevant information must be available for renewal to operate. Temporal effects on such processes also may be independent or secondary to interactional factors (such as the number of items to be rehearsed in a specified time).

Temporal and interactional processes, which may or may not affect each other as well as the previously considered factors. More specific formulation of such processes will depend in part on conceptions of the organization of STM, embodying resolution of problems such as what constitutes an event in STS (in contrast to an event as presented or retrieved), in what form is information about events encoded in STS, do temporal and interactional processes affect individual independent items in STS or the contents of STS as a whole, do temporal processes operate on an event over its whole time in storage or only over the interval before the next presentation, and is interference generated by items individually or collectively? Commonly, these processes are thought of in a more restricted sense as decay and interference, which are often considered as opposed alternatives. In studies of STM such as this one, which seem to require "verbatim" retention of (information about) discrete independent events, decay and interference may be viewed as applying to specific events independently stored. Decay then refers to a "passive," autonomous loss of information over time without necessary reference to other items in storage, and interference to an "active" loss or decrease in accessibility of information about some item due to the presence of one or more other items in STS.

Both proactive and retroactive interference have been shown in STM for single letters by Wickelgren (1966), replicating Murdock's demonstration of PI in STM (Murdock, 1961). Wickelgren distinguished between associative interference (which is a type of retrieval interference due to establishment of competing associations) and storage interference (which reduces the strength of the correct association), and suggested the need for a two factor theory of forgetting in STM: associative interference and decay (or storage interference). Both PI and RI presumably might be either specific or non-specific with respect to an event encoded and retained in STS. The former refers to competition among certain events for some specific storage form or space, and the latter to competition among any events for non-specific storage space, form or processing time.

Whether decay and interference (or for that matter, perception, encoding, and renewal processes as well) apply to information about individual items or to the contents of STS as a whole may depend on the situation under consideration: the former may apply when retention of information about individual items alone is required and the latter when information about the sequence as a whole is required. For the former case, one reasonable way to approach relative retention of information about sequentially presented events is to consider factors affecting the relative strength (value) of such information at time of readout. These may include: initial value, rate of rise to maximum value, maximum value, and rate of decline from maximum value. These in turn may be affected by temporal factors alone, by mutual interaction with other items alone, or by both. Temporal factors include time before arrival of the next item to be processed, processing time, and time before readout. Interactional factors include array size, number of items preceding or following, and order of arrival (serial position), as well as relations among items such as similarity. Temporal and interactional factors may themselves interact so that interaction of items may be a function of temporal factors, and rate of increase or decrease of item strength may be affected by interactional factors. Although the missing scan requires retention of information about the occurrence of all items presented so as to choose the missing item correctly, it is probably reasonable to consider that the information encoded in this case refers to the specific discrete items presented, since the missing scan requires information about all items individually but not as related to each other in an organized whole.

Reorganization and recoding in STS is suggested both 
by consideration of possible functions of STM (Bower, in Hilgard \& Bower, 1966, pp. 504-505) and by experiments on STM for sentences (Savin \& Perchonock, 1965). Even for "verbatim" recovery of specific discrete events, information about individual events may be recoded so that their form of storage is no longer independent of other items but rather is predicated in terms of the organization of larger groups (e.g., chunking: Miller, 1956) or even the whole contents of STS. In this case decay and interference might refer more naturally to the organized contents of STS as a whole, eventuating secondarily in loss of (certain) information about specific discrete events as presented.

Which factors are most relevant here may be suggested by consideration of the major findings of this study. The linear increase in total errors as rate of presentation increases might be due to impairment of perception and encoding, lower strength of traces (less consolidation), impairment of recoding and reorganization of contents of STS, or impairment of renewal processes. This finding seems incompatible with the notion of decay as a major factor in STS since decreased time for decay should result in fewer errors when time before readout is decreased (although it is possible that rate of decay might be greater when strength at onset of decay is lower, or that renewal processes opposing decay are less effective at faster rates of presentation). The most plausible basis for this finding seems to be decreased perception, encoding, or strength level, which, however, might be secondary to impaired reorganization of STS if the current state of organization prescribes the kind of information about a presented event relevant for these processes.

The increasing primacy effect found at faster rates of presentation could reflect decreased decay, since the decrease in time for decay before readout is greatest for earliest presented and least for most recently presented items. However, decreased (retroactive) interference during less time before readout (possibly by fewer subsequent items of lesser strength secondary to impaired perception, encoding, or growth of trace strength) could also account for the primacy effect. In addition, the notion of decreased interference could also account for the finding that a primacy effect is seen (Fig. 1) at the slow presentation rate of $1 / \mathrm{sec}$. when partial (.25 sec.) presentation duration is used, but not when full (1. sec.) presentation duration is used, while decay could not Furthermore, since the increase of total errors at faster rates of presentation is due to an increase of errors in the more recently presented items of the sequence not subject to the primacy effect, it might be argued that this increase is secondary to (proactive) inhibition by earlier items. In other words, interference might account for both the primacy effect and the increase in errors as rate of presentation increases and time in STS before readout decreases, on the basis of a shift from retroactive to proactive interference due to increased time for proactive interference effects to dissipate (or be overcome by $\mathrm{RI}$ ) and/or for retroactive interference effects to accumulate (or operate effectively). In any case, the primacy effect would mean that interference is time dependent.

For a view of STS undergoing active reorganization of the whole contents of STS, a parallel account of the findings may be considered. At slow rates of presentation there is enough time for reorganization (and recoding) of the current contents of STS in terms of criterial attributes relevant to retention of the most recently presented item. In the course of such a retroactive reorganization of STS contents in terms of subsequent items, information about earlier items would be subject to more recoding and loss than that about later items, leading to a serial position error curve of the form found. As rate of presentation increases, there would be less time for reorganization of STS contents in terms of the currently presented item before the next item arrives, so that earlier items would benefit from decreased (retroactive) recoding, yielding a primacy effect, while later items would suffer from the overall decrease in (effective) reorganization, resulting in an increase of errors. In other words, less effective reorganization at faster rates of presentation would increase the loss of information and thus the probability of error, but the earliest items would benefit from such impairment of serial recoding because the organization of STS contents that did exist would be predicated more in terms of information relevant to their retention and subject to decreased attrition by subsequent reorganization. This view might similarly account for the finding of a primacy effect at slow rates with partial (.25 sec.) presentation duration.

The form of the error distribution at the slow $1 / \mathrm{sec}$. presentation rate with full duration presentation could reflect interference (RI), decay, or retroactive reorganization of STS contents. The finding that the error distribution remains linear with the same slope over at least the most recently presented half of sequence argues against decay since increasing rate of presentation would be expected to lead to retention of significant linearity over the entire error distribution with slope changing toward zero, because the relative differences in storage time before readout for sequentially presented items decrease as rate of presentation increases. This finding is compatible with interference by some interactional factor such as the number of presentations in a sequence, the number of alternatives that may be presented (array size), the number of items preceding or following, or the arder of arrival (serial position) which remains constant at different rates of presentation. The uniform 
increase of errors which leads to retention of the same slope with increasing rate of presentation also indicates that interference is time dependent and must operate over some time period whose relative change is the same for different serial positions as rate of presentation increases. Two temporal parameters which remain constant despite increase in rate of presentation are the relative (\%) decrease in storage time before readout, and the relative $(\%)$ decrease in time before arrival of the next item. Either of these two might result in the same relative strength (or probability of retention) or items in different serial positions at different rates of presentation. The former would seem to apply best if interference or reorganization of information about an item operated over the entire time in storage, while the latter seems more pertinent if those processes operate only during the interval between presentations. Of the two, time in STS before readout seems more relevant since it decreases from first through last presented items.

The temporal effects in storage described here appear most compatible with the operation primarily of time dependent interactional factors sich as interference or reorganization in STS. While decay might account for the error distribution at $1 / \mathrm{sec}$. and for the primacy effect with increasing rate of presentation, the increase in total errors due to increase of errors over at least the last half of the sequence together with persistence of the same slope of the linear component at faster rates argue against decay. The best counterargument would seem to be that at faster rates there is less time for rehearsal, which is devoted principally to earlier items and generates a primacy effect, but this still would not convincingly account for the persistence of linearity with the same slope. Furthermore, the joint operation of decay and rehearsal should lead to decreased relative differences in strength of items resulting in change of slope toward zero. Finally, it is not clear why decrease of time before readout should necessarily reduce the effectiveness of renewal processes such as rehearsal in offsetting decay. The effectiveness of renewal must depend at least in part on the information available at time of renewal, and this may be better when there has been less decay, so that, for instance, there may be less erroneous rehearsal of incorrect items. It would seem that the reciprocal relations of decay and rehearsal must be explicated in a way that clearly indicates why rehearsal should be less effective as rate of presentation increases, before impaired rehearsal can convincingly be invoked to account for increased errors when there is less time for decay.

Perceptual and encoding processes might generate the error distribution at the slow rate and account for the increase in errors, but cannot by themselves explain the primacy effect. Consolidation (or rehearsal) might account for the increase in errors and the primacy effect, but not for the direction of the error distribution since it would predict an increase of errors with increasing recency, rather than the decrease which was found.

While interference by itself might account for all the findings, the increase of errors probably requires the implication of decreased perception and encoding, although this might possibly be secondary to interference. Thus, at slow rates of presentation (1/sec.) perception and encoding are effective and the error distribution is primarily due to RI, any PI being overcome by time and/or RI. As the rate of presentation increases, perception and encoding of earlier items is effective, but that of later (more recent) items is impaired because perception and encoding may depend on the alternatives relevant at each sequential presentation and the appropriate reduction in number of alternatives is impaired at faster rates. Such a possibility is suggested by the finding of decreased memory span for mixed letters and digits (Conrad, 1962) and the superiority of digit over letter span of apprehension as well as the lowered span of apprehension for mixed letters and digits, which have been discussed in terms of transitional probabilities and their possible relation to "chunking" (Warrington et al, 1966). Whether the increase of errors comes about in this fashion or simply because of insufficient time for perception and encoding at faster rates of presentation, a primacy effect emerges because there is less RI, either because later items are less likely to be encoded or are less adequately encoded or both. As indicated, reorganization and recoding in STS may also account for these findings. Whether there is a real difference between interference and recoding, or whether both are time dependent interactional factors applying respectively to individual items and the whole contents of STS remains to be seen.

In conclusion, this study provides evidence for the operation of time dependent interactional factors in short-term storage, by a method (the missing scan) which requires storage of all items presented and evaluation of the relative retention of all concurrently without further interference after presentation by testing or retrieval effects. The results urge further study of relative retention in STS by other retrievalfree methods to clarify the operation of relevant interactional factors.

\section{References}

Brown, J. Some tests of the decay theory of memory. Quart. J. erp Psychol., 1958, 10, 12-21.

Buschke, H. Retention in immediate memory estimated without trieval. Science, 1963a, 140, 56-57.

Buschke, H. Relative retention in immediate memory determined by the missing scan method. Nature, $1963 \mathrm{~b}, 200,1129-1130$.

Conrad, $\mathbf{R}$. Practice, familiarity and reading rate for words and nonsense syllables. Quart. J. exp. Psychol., 1962, 14, 71-76. 
Conrad, R., \& Hille, B. A. The decay theory of immediate memory and paced recall. Canad. J. Psychol., 1958, 12, 1-6.

Corballis, M.D. Memory span as a function of variable presentation speeds and stimulus durations. J. exp. Psychol., 1966, 71, 461465.

Crowder, R. G. Visual presentation of stimuli in immediate memory. Psychon. Sci., 1966, 6, 449-450.

Hilgard, E. R., \& Bower, G. H. Theories of learning. New York: Appleton-Century-Crofts, 1966 (3rd edition).

Mackworth, J. F. Presentation rate and immediate memory. Canad. J. Psychol., 1962, 16, 42-47.

McNemar, Q. Psychological statistics. New York: Wiley, 1962 (3rd edition).

Miller, G. A. The magical number seven, plus or minus two: some limitations on our capacity for processing information. Psychol. Rev., 1956, 63, 81-97.

Murdock, B. B., Jr. The retention of individual items. J. exp. Psychol., 1961, 62, 618-625.

Noman, D. A. Acquisition and retention in short-term memory. $J$. exp. Psychol., 1966, 72, 369-381.

Peterson, L. R. Short-term verbal memory and learning. Psychol. Rev., 1966, 73, 193-207.
Posner, M. I. Immediate memory in sequential tasks. Psychol. Bull., 1963, 60, 333-349.

Savin, H. B., \& Perchonock, E. Grammatical structure and the immediate recall of English sentences. J. verbal Learn. verbal Behav., 1965, 4, 348-353.

Warrington, E. K., Kinsboume, M., \& James, M. Uncertainty and transitional probability in the span of apprehension. Brit. J. Psychol., 1966, 57, 1 and 2, 7-16.

Waugh, N. C., \& Norman, D. A. Primary memory. Psychol. Rev. $1965,72,89=104$.

Wickelgren, W. A. Phonemic similarity and interference in shortterm memory for single letters. J. exp. Psychol., 1966, 71, 396-404.

\section{Notes}

1. This research was supported by USPHS Research Grant MH08556 and USPHS Research Career Development Award K3-MH-23, 796 to Herman Buschke from the National Institute of Mental Health. 2. We thank Dr. J. Chase, Stanford University Department of Statistics, for statistical assistance.

(Accepted for publication January 2, 1967.) 